\title{
STABILITY THEORY OF DISSIPATIVE TRAPPED-ELECTRON AND TRAPPED-ION MODES
}

BY

W. HORTON, JR, , D, W. ROSS, W. M. TANG, $H$. L. BERK,

E. A, FRIEMAN, R, E, LAQUEY

R. V. LOVELACE, S. M. MAHAJAN, M. $N$, ROSENBLUTH AND P. H. RUTHERFORD

\section{PLASMA PHYSICS LABORATORY}

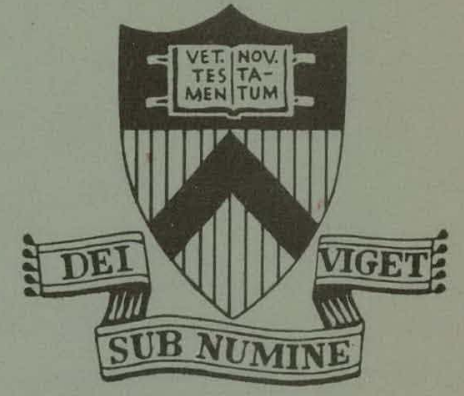

\section{PRINCETON UNIVERSITY PRINCETON, NEW JERSEY}

This work was supported by U. S. Atomic Energy Commission Contract AT (11-1) - 3073. Reproduction, translation, publication, use, and disposal, in whole or in part, by or for the United States Covernment is permitted. 


\section{DISCLAIMER}

This report was prepared as an account of work sponsored by an agency of the United States Government. Neither the United States Government nor any agency Thereof, nor any of their employees, makes any warranty, express or implied, or assumes any legal liability or responsibility for the accuracy, completeness, or usefulness of any information, apparatus, product, or process disclosed, or represents that its use would not infringe privately owned rights. Reference herein to any specific commercial product, process, or service by trade name, trademark, manufacturer, or otherwise does not necessarily constitute or imply its endorsement, recommendation, or favoring by the United States Government or any agency thereof. The views and opinions of authors expressed herein do not necessarily state or reflect those of the United States Government or any agency thereof. 


\section{DISCLAIMER}

Portions of this document may be illegible in electronic image products. Images are produced from the best available original document. 
Stability Theory of Dissipative * Trapped-Electron and Trapped-Ion Modes

W. Horton, Jr, and D. W. Ross

Fusion Research Center, University of Texas at Austin Austin, Texas 78712 USA

W. M. Tang, H. L. Berk, ${ }^{\dagger}$ E. A. Frieman, R. E. LaQuey

R. V. Lovelace, S. M. Mahajan, M. N. Rosenbluth $\neq$ and $P . H$. Rutherford

Princeton University, Plasma Physics Laboratory

Princeton, New Jersey 08540 USA

\section{ABSTRACT}

This paper treats topics in the linear theory of dissipative trappedelectron instabilities, and in the nonlinear theory of the dissipative trapped-ion instabilities. Stability criteria are obtained for dissipative trapped-electron modes in a tokamak-type magnetic field with shear. In the important case where the mode is driven by the electron temperature gradient, and neglecting "ballooning" effects, shear is found to stabilize the mode if typically $\mathrm{r}_{\mathrm{n}} / \mathrm{L}_{\mathrm{s}}>0.1(\mathrm{r} / 2 \mathrm{R})^{1 / 2} \mathrm{~d} \ln \mathrm{T}_{\mathrm{e}} / \mathrm{d} \ell \mathrm{nn}$, a condition that can be attained. With weak shear, it is shown, however, that the mode-structure along the field can exhibit substantial "ballooning." The dissipative trapped-ion mode, which is not shear-stabilized, is considered in its nonlinear regime. Including nonlinear $\underline{E} \times \underline{B}$ motions, exact stationary trappedion-mode solitary-wave solutions are found, in which the wave energy in longer azimuthal wavelengths passes to shorter-wavelength components that are strongly damped by ion bounce resonances; the amplitudes are sufficiently reduced that a diffusion lower than Kadomtsev's is indicated. As a second nonlinear mechanism, anomalous velocity-space scattering due to the development of an unstable loss-cone-type ion distribution function is considered; this mechanism raises the effective ion collision frequency and increases the stability of the longer azimuthal-wavelength modes.

Trapped-Electron Mode

In the collisionless regime, and in the frequency range between the thermal ion and thermal electron bounce frequencies $\omega_{b}=2 \pi / \phi \mathrm{ds} / v_{11}$, for a tokamak system the drift wave occurs as a trapped-electron mode [1] driven unstable by the collisional detrapping of the electron during a wave period. The mode is electrostatic with the fluctuating potential $\phi(\underset{x}{x}, t)=\phi_{o}(r) P(\theta) \exp (i m \theta-i \ell \zeta)$, where $\theta$ is the angle the short way 
and $\zeta$ the angle the long way around. the torus. Here we first consider the radially localized modes that occur about the high-order rational surfaces $m=\ell q\left(r_{i}\right)$ with $m$, $\ell$ integral. We treat the ballooning in $\theta$ as a higher order effect and eliminate the $P(\theta)$ dependence from the mode equation. In cases where the shear is sufficiently weak, the ballooning which arises from the trapped electron integral operator appears as a first-order effect and gives rise to a different class of modes which will be discussed later. The radially localized modes occur with width of order $\Delta r=\rho / s^{1 / 2}$ where $\rho=c\left(m_{i} T_{e}\right)^{1 / 2} / e B$, and the shear parameter is given by $S=r_{n} / L_{s}=(\varepsilon / q) \times$ $|d \ln q(\mathrm{r}) / \mathrm{d} \ln \mathrm{n}(\mathrm{r})|$. The condition that the mode frequency be greater than the thermal ion bounce frequency requires that $k_{1} \rho>\left(r_{n} / L_{c}\right)\left(T_{i} / T_{e}\right)^{1 / 2}$. The electron distribution function is calculated for general $\omega / \omega_{b e}^{T}$ and $\nu_{e}^{*}=\nu_{e}^{e f} / \omega_{b e}^{T}$ by introducing the following approximations. First the elliptic-function bounce orbits in the field $B=B_{0}(1-\varepsilon \cos \theta)$ are approximated by their sinusoidal limit $\theta(t)=\theta_{0}(\lambda) \sin \left[\omega\left(t-t_{0}\right)\right]$, where $\theta_{0}(\lambda)=$ $\left[2\left(1-\lambda \mathrm{B}_{\min }\right) / \varepsilon \lambda \mathrm{B}_{\mathrm{o}}\right]^{1 / 2}$ and $\omega_{\mathrm{b}}^{\mathrm{T}}=\left(\varepsilon \mathrm{E} \lambda \mathrm{B}_{\mathrm{o}}\right)^{1 / 2} / \mathrm{qR}$ with $\mathrm{B}_{\max }^{-1}<\lambda<\mathrm{B}_{\min }^{-1}$ defining the trapped particles. We note, however, that for the trapped orbits to cover the full range $0 \leq \theta_{0} \leq \pi$ we must lower $\lambda_{\min } B_{0}$ from $1 /(1+\varepsilon)$ to $1 /\left[1+\varepsilon\left(\pi^{2} / 2-1\right)\right]$ in this model. The second approximation is to write for the dominant collisional process the local trapped electron collision operator $C\left(\tilde{f}_{e}^{T}\right)=-v_{e}^{e f f}\left(v_{e} / v\right) 3^{\tilde{f}_{e}^{T}}$ with $v_{e}^{\text {eff }}=v_{e i}\left(1+z^{e f f}\right) / \varepsilon$, as introduced by Kadomtsev and Pogutse [1] and shown in our work to give results in good agreement with more complete calculations based on the FokkerPlanck operator [2]. Within this mode 1 the trapped electron density $\tilde{\mathrm{n}}_{\mathbf{e}}^{\mathrm{T}}$ is readily calculated as $\tilde{n}_{e}^{T} / n_{0}=-(\varepsilon / 2)^{1 / 2}\left(e \tilde{\phi} / T_{e}\right) \sum Y_{n}(\omega)$ where

$$
\left(\frac{\varepsilon}{2}\right)^{1 / 2} Y_{n}(\omega)=\left\langle\frac{\omega-\omega_{*_{e}}^{T}(E)}{\omega-n \omega_{b e}^{T}+i \nu_{e}^{e f f}(E)} J_{n}^{2}\left(|m-\ell q| \theta_{0}\right)\right\rangle
$$

where $\omega_{* e}^{T}=\omega_{* e}\left[1+n_{e}\left(E / T_{e}-3 / 2\right)\right], n_{j}=d \ln T_{j} / d \ell n n_{0}$, and $<>$ denotes the average over energies and pitch angles of the trapped electrons. For $k_{11} L_{c}=|m-l q| \ll 1$ we recover from Eq. (1) the well-known low frequency limit for $\tilde{n}_{e}^{T}$, and for $k_{11} L_{c}=|m-\ell q| \gg>1$ and $\omega>\omega_{b}^{T}$ we recover from Eq. (1) the slab approximation result $\operatorname{Im} \tilde{n}_{e}^{T} \propto \pi^{1 / 2}\left[\omega-\omega_{* e}\left(1-n_{e} / 2\right)\right] /\left|k_{n}\right| v_{e}$, for which the critical shear and growth rates are well known [3]. In applying Eq. (1) we further approximate the $\lambda$-integral and carry out the energy integrals numerically, since the strong energy dispersion of $\nu_{e}^{\text {eff }}(E)$. is an essential feature of the dissipative phenomena. In Fig. (1) the dissipative part of $I=\operatorname{Im} Y_{o}\left(\omega=\omega_{\star} e^{\prime}\right) / n_{e}$ is shown at $m=\ell q$ and compared with the asymptotic expansion of the Fokker-Planck result to the first order, $I_{\mathrm{F}}(\mathrm{I})$, and to the third order, I $(3)$. The equivalent asymptotic expansions of I are also shown and are seen to agree closely with the Fokker-Planck expansions. Note the substantial shift to larger $\omega_{* e} / \nu_{e}^{\text {eff }}$ and the increase in the value of the maximum of I compared to the asymptotic approximations.

For mode frequencies greater than the circulating ion transit frequency $\left(\omega>v_{i} / q R\right)$, orbit periodicity effects are negligible, and the perturbed ion density $\tilde{n}_{i}$ is calculated as in the slab model to obtain $\tilde{n}_{i}$ as a function of $\mathrm{Z}(\zeta)$ and $\Gamma_{0,1}=\exp (-b) I_{0,1}(b)$, where $b=k_{1}^{2} \rho_{i}^{2} / 2$, and $\zeta=$ $\omega /\left|k_{11}\right| v_{i}$, in which the local waye numbers are given by $k_{11}=(m-l q) / L_{c}$ and $k_{\perp}=\mathrm{m} / \mathrm{r}$. Here, $\mathrm{z}(\zeta)=\pi^{-1 / 2} \int_{-\infty}^{+\infty} \mathrm{dx} \exp \left(-\mathrm{x}^{2}\right) /(\mathrm{x}-\zeta-\mathrm{i} \delta), \mathrm{v}_{j}=$ $\left(2 \mathrm{~T}_{j} / \mathrm{m}_{j}\right)^{1 / 2}$ and $\rho_{j}=v_{j} / \omega_{c j}$. By invoking quasi-neutrality we obtain the mode equation for the fluctuating potential. In this section, we consider the radial dependence of the mode equation and eliminate the $\theta$-dependence 
by contracting with the $\theta$-adjoint function $[g(q R d \theta / B) P * \exp (-i m \theta+i l \zeta)]$. Below, the $\theta$-dependence is considered in a complementary calculation.

The equation we obtain is

$$
\begin{aligned}
& p^{2} \frac{\partial^{2} \phi_{o}}{\partial r^{2}}+\left\{-\zeta Z\left[1-\frac{\omega_{* i}}{\omega}\left(1-\frac{1}{2} \eta_{i}\right)\right] \frac{d \Gamma_{o}}{d b}+\zeta z \frac{\eta_{i} \omega_{* i}}{\omega} \frac{d}{d b}\left[b\left(\Gamma_{1}-\Gamma_{o}\right)\right]+\zeta^{2}(1+\zeta Z) \frac{\eta_{i} \omega_{*_{i}}}{\omega} \frac{d \Gamma_{o}}{d b}\right\}^{-1} \\
& \left\{\tau\left[1+\zeta Z\left(1-\frac{\omega_{*_{i}}}{\omega}\left(1-\frac{1}{2} \eta_{i}\right)\right) \Gamma_{0}-\zeta Z \frac{\eta_{i} \omega_{*_{i}}}{\omega} b\left(\Gamma_{1}-\Gamma_{0}\right)-\zeta^{2}(1+\zeta Z) \frac{\eta_{i} \omega_{*_{i}}}{\omega} \Gamma_{0}\right]\right. \\
& \left.+1-\left(\frac{\varepsilon}{2}\right)^{1 / 2} \sum_{n=-\infty}^{+\infty} Y_{n}\right\} \phi_{0}=0 \text {, }
\end{aligned}
$$

where $\omega_{* j}=\left(c k_{1} T_{j} / e_{j} B\right) d \ell n n / d r$, and $\tau=T_{e} / T_{i}$. Equation (2) describes modes localized to the rational surfaces where ion Landau damping is exponentially small. In the vicinity of the rational surface Eq. (2) reduces to $\phi^{\prime \prime}+\left[Q_{0}(\omega)+s^{2} x^{2} Q_{1}(\omega)\right] \phi=0$, and has finite, outgoing energy solutions for $\omega=\omega_{0}+i \gamma_{k}$, where

$$
\omega_{0}=\omega_{*_{e}} \Omega_{0}=\frac{\omega_{*_{e}}\left[\Gamma_{0}+n_{i} b\left(\Gamma_{1}-\Gamma_{0}\right)\right]}{1+\tau\left(1-\Gamma_{0}\right)} \text {, }
$$

and

$$
\begin{aligned}
& \gamma_{k}=\frac{\omega_{* e} \Omega_{0}^{2}}{\Gamma_{0}+n_{i} b\left(\Gamma_{1}-\Gamma_{0}\right)}\left\{\left(\frac{\varepsilon}{2}\right)^{1 / 2} \operatorname{Im} Y_{0}\left(\omega_{0}\right)\right. \\
& -\frac{\mathrm{s}}{\Omega_{0}}\left|\frac{\mathrm{d} \Gamma_{\mathrm{o}}}{\mathrm{db}}\right|^{1 / 2}\left[1+\frac{1+n_{i}}{\Omega_{0} \tau}\right]^{1 / 2}\left[1+\frac{1}{\Omega_{0} \tau}\left(1+n_{i}\left(1-\frac{b\left(2 \Gamma_{0}-5 \Gamma_{1} / 2\right.}{\Gamma_{0}-\Gamma_{1}}\right)\right]^{1 / 2}\right. \\
& \left.\left[1-\frac{\Omega_{0}^{2} L_{c}^{2}\left(k_{\perp} \rho\right)^{2}}{\Gamma_{0} r_{n}^{2}} \frac{(\varepsilon / 2)^{1 / 2}\left(Y_{o} / 2-Y_{1} / 4\right)}{1+\left(1+\eta_{i}\right) / \Omega_{o} \tau}\right]^{1 / 2}\right\}
\end{aligned}
$$

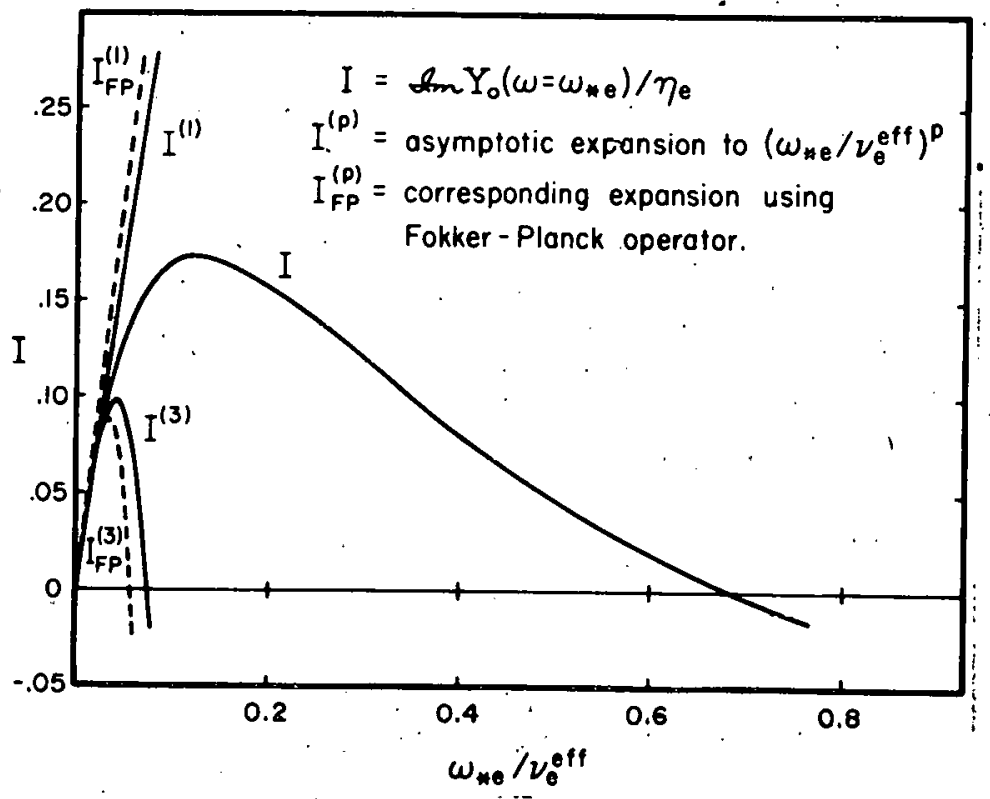

Fig. 1.

The dominant trapped electron destabilizing term for positive $n_{e}$. The values obtained from the collisional model are denoted by $I$, the asymptotic expansions by $I(p)$, and the corresponding Fokker-Planck expansions by $I_{\mathrm{FP}}(\mathrm{p})$. 
In examining the growth rate $\gamma_{k}$ we first observe that there are two regimes, $k_{1} \rho \gtrless K_{c}$, where $K_{c}$ is approximately independent of machine size when expressed in terms of $\nu_{e}^{\star}$ and is given by $k_{c}=0.7\left(r_{n} / L_{c}\right)\left(\varepsilon m_{i} / m_{e}\right) 1 / 2 \nu_{e}^{*}$. For $k_{\underline{1}} \rho<K_{c}$ the normal electron temperature gradient $\left(n_{e}>0\right)$ is destabilizing, and for $k_{t} \rho>k_{c}$ this gradient is stabilizing. For $v_{e} \leq 1$ we have $\mathrm{K}_{\mathrm{c}}<1$ for typicali system parameters, and if we restrict the consideration to $\tilde{k}_{1} \rho_{i}<1$; arguing that such modes maximize the diffusion then we have

$$
\begin{gathered}
\gamma_{k} \simeq \omega_{*_{e}}\left\{\left(\frac{\varepsilon}{2}\right)^{1 / 2}\left[n_{e} \mathrm{y}_{1}\left(k_{\perp} \rho / \mathrm{K}_{c}\right)+k_{\perp}^{2} \rho^{2}\left(1+\frac{1+\eta_{i}}{\tau}\right) y_{2}\left(k_{\perp} \rho / k_{c}\right)\right]\right. \\
\left.-s\left(1+\frac{1+\eta_{i}}{\tau}\right)\left[1-\left(\frac{L_{c}}{r_{n}}\right)^{2} \frac{\left(k_{\perp} \rho\right)^{2}(\varepsilon / 2)^{1 / 2}\left(Y_{o} / 2-Y_{1} / 4\right)}{1+\left(1+n_{i}\right) / \tau}\right]^{1 / 2}\right\},
\end{gathered}
$$

where $y_{j}(x)$ and $y_{2}(x)$ are the coefficients in the dissipative parts of $Y_{0}$ proportional to $\eta_{e}$ and the frequency shift, respectively. For $k_{1} \rho<k_{c}$, $\mathrm{R}_{e} \mathrm{Y}_{\mathrm{o}} \simeq-0.82\left(\mathrm{k}_{\underline{1}} \rho / \mathrm{K}_{\mathrm{c}}\right)$, and the radial anti-well steepening due to $\mathrm{J}_{0}^{2}\left(\mathrm{k}_{1}, \mathrm{~L}_{\mathrm{c}}\right)$ is small if $k_{1} \rho<\left(r_{n} / L_{c}\right)\left(m_{i} / m_{e}\right) 1 / \delta v_{e}^{* 1 / 3}$. The critical shear required for stability in this regime is approximately given by

$$
s_{\text {crit }}=\left(\frac{\varepsilon}{2}\right)^{1 / 2}\left[\frac{0.17 n_{e}+0.007 \mathrm{k}_{c}^{2}\left(1+\left(1+n_{i}\right) / \tau\right)}{1+\left(1+n_{i}\right) / \tau}\right]
$$

and for $S \ll S_{\text {crit }}$ and $n_{e} \sim 1$ the maximum growth rate occurs at $k_{1} \rho=0.44 K_{c}$ and is given by $\gamma_{\max }=0.025 \varepsilon^{1 / 2} n_{e} \nu_{e}^{e f f}$. As the estimate for the anomalous diffusion rate we obtain from $\gamma_{\max } / k_{r}^{2}$ that

$$
D_{A}=0.025 \varepsilon^{1 / 2} n_{e}\left(L_{s} / r_{n}\right) \nu_{e}^{e f f} \rho^{2} \cdot=c_{2} \nu_{e i} \rho_{e \theta}^{2}
$$

where $c_{2}=0.025 \eta_{e} \varepsilon^{1 / 2}\left(m_{i} / m_{e}\right)|d \ell n n / q d \ell n q|$ and $\rho_{e \theta}=\rho_{e} q / \varepsilon$. The diffusion $D_{A}$ differs by a factor $q$ and a numerical coefficient from that given earlier by Galeev [2]. The scaling of $D_{A}$ is similar to the neoclassical diffusion coefficient $D_{\text {neo }}$ and $D_{A} / D_{\text {neo }}=c_{2} / \varepsilon^{1 / 2} \approx 50-80$. This diffusion coefficient $\mathrm{D}_{\mathrm{A}}$ and the associated electron thermal conductivity $\mathrm{K}_{\mathrm{A}}=$ $(7 / 2) D_{A} / n_{e}$ are used in the Princeton anomalous transport code which predicts loss rates not inconsistent with Tokamak experiments. We also remark that the $n(r), T_{e}(r)$ and $q(r)$ profiles reported for ST are consistent with being near a state of marginal stability given by Eq. (5), further suggesting the'importance of the mode for the experiments.

Further into the banana regime $K_{c} \ll 1$, and fast growing modes with $k_{1} \rho>k_{c}$ occur, driven by the cross-field ion inertia effect and stabilized by positive $n_{e}$. In this case the steepening of the anti-well by $\mathrm{J}_{0}^{2}\left(\mathrm{k}_{1,} \mathrm{~L}_{\mathrm{c}}\right)$ is important except for $k_{1} \rho<2\left(r_{n} / L_{c}\right) \varepsilon^{-1 / 4}$. Although the most dangerous modes here have $k_{\perp} \rho>2\left(r_{n} / L_{c}\right) \varepsilon^{-1} / 4$, we estimate that, with the radial-well enhancement, the critical shear in this regime is

$$
\mathrm{s}_{\text {crit }} \simeq\left(\frac{\varepsilon}{2}\right)^{1 / 2}\left[0.2-\frac{0.13 \cdot n_{e}}{1+\left(1+n_{i}\right) / \tau}\right] /\left[1+0.04 \varepsilon^{1 / 2}\left(\frac{L_{c}}{r_{n}}\right)^{2} \frac{1+0.8 n_{e}}{1+\left(1+n_{i}\right) / \tau}\right]^{1 / 2} .
$$

For $S \ll S$ crit the maximum growth rate is given by $\gamma_{\max } \simeq 0.06 \varepsilon^{1 / 2} \nu_{\mathrm{e}}$, and the diffusion implied is $D \simeq 0.06 \varepsilon^{1 / 2} v_{e} \rho^{2}\left(L_{s} / r_{n}\right)$ with $\operatorname{maxD}=$ $0.06 \varepsilon^{1 / 2}\left(\rho / r_{n}\right)\left(L_{s} / r_{n}\right)\left(c T e^{/ e B}\right)$. 
In the above radial eigenmode calculations the usual drift-wave-type analysis is followed with the integral electron-collision term (the $Y_{n}$ ) treated as perturbations in the mode equation. We find that for sufficiently small shear there is also a class of growing modes which have a substantial "ballooning" component along the magnetic field. Here the mode structure is determined by the integral electron-collision term rather than by the ion sound propagation term, and the basic equation has the form:

$$
\left[A \frac{\partial^{2}}{\partial \theta^{2}}+B+\frac{\rho_{i}^{2}}{2}\left(\frac{T_{e}}{T_{i}}+\frac{\omega_{*_{e}}}{\omega}\right)\left(k_{r}+\ell q^{\prime} \theta\right)^{2}\right] \phi=D \bar{\phi}
$$

where $A=\left(\frac{T_{e}}{T_{i}}+\frac{\omega_{*_{e}}}{\omega}\right) \frac{v_{i}^{2}}{2 \omega^{2} q^{2} R^{2}}, \quad B=1-\frac{\omega_{*_{e}}}{\omega}+\left(\frac{T_{e}}{T_{i}}+\frac{\omega_{*_{e}}}{\omega}\right) \frac{k_{\perp}^{2} p_{i}^{2}}{2}$,

$D$ (integral operator) $=\int d^{3} v F_{e}^{M}\left(\frac{\omega-\omega_{\star}^{T}}{\omega+1 \nu_{e}^{e f f}}\right), \phi=\hat{\phi} \exp [-i \ell(\zeta-q \theta)]$,

$\phi=\Phi \mathrm{ds} \hat{\phi} / v_{11} / \oint \mathrm{ds} / v_{11}, k_{1}=\ell q / r$, and $\theta$ is the poloidal angle measured from the outside of the torus.

Treating the sound term (A) and the shear term (third term) as small, and noting that to lowest order $\omega=\omega_{*}$, we see that Eq. (8) reduces to the same type of integral equation encountered in solving the dissipative trapped-ion mode eigenfunction problem. Application of a variational procedure then yields a solution for the orbit-averaged potential $\Phi$ which maximfies the destabilizing term on the right side of this equation. The resultant growth rate is $\gamma \simeq\left(\omega_{0}^{2} / \omega_{* e}\right)(\varepsilon / 2)^{1 / 2}$ Im $Y_{0}$, and the corresponding form of $\bar{\phi}$ can be inverted to give $\hat{\phi} \simeq \cos (\theta / 2)$. However, this lowest order solution does not satisfy the requirements that the potential $\phi$ and its $\theta$ derivative be continuous at $\theta= \pm \pi$. It is thus necessary to include the first term in Eq. (8) and solve the resultant differntial equation as a boundary layer problem in the region around $\theta= \pm \pi$ where the lowest order solution breaks down. Here it is found that the eigenvalue (and hence the growth rate) is not appreciably affected by this modification but that the mode now has the structure

$$
\hat{\phi}(\theta)=\cos \frac{\theta}{2}+\frac{\delta}{2} \exp \left(-\frac{\pi}{\delta}\right)\left[\cosh \left(\frac{\theta}{\delta}\right)+\cosh \left(\frac{\theta}{\delta}+2 \pi i \ell q\right)\right]
$$

where $\delta \equiv i(A / D)^{1 / 2}$. This solution satisfies the required boundary conditions and is valid provided $\left(r_{n} / L_{c}\right)\left(1.5 / n_{e}^{1 / 2} \varepsilon^{1 / 4}\right)<k_{1} \rho_{i}<\left(r_{n} / L_{c}\right)\left(\varepsilon^{1 / 4} n_{e}^{1 / 2 / 5 S}\right)$.

If a step model for the $\theta$-dependence of the magnetic field is adopted, then the preceding weak shear problem can be solved without recourse to the perturbation treatment. Since a narrow boundary layer is no longer required, the lower constraint can be removed. The solution takes the form

$$
\begin{gathered}
\hat{\phi}(\theta)=\hat{c}\left\{[1-\exp (4 \pi i \ell q)] \sin (\alpha \pi) \sin (\alpha \theta)-[1-\exp (2 \pi i \ell q)]^{2} \cos (\alpha \pi) \cos (\alpha \theta)\right. \\
\left.+[1+\exp (2 \pi i \ell q)]^{2} \sin ^{2}(\alpha \pi)+[1-\exp (2 \pi i \ell q)]^{2} \cos ^{2}(\alpha \pi)\right\},
\end{gathered}
$$

where $\hat{c}=\bar{\phi} \frac{D}{B}\left\{[1+\exp (2 \pi i l q)]^{2} \sin ^{2}(\alpha \pi)+[1-\exp (2 \pi i \ell q)]^{2} \cos ^{2}(\alpha \pi)\right\}$ $\alpha \equiv(B / A) 1 / 2$, and the corresponding dispersion relation determining the eigenvalue $B$ is 


$$
\frac{B}{D}=1-\frac{1}{\alpha \pi}\left[\frac{\sin (\alpha \pi) \cos (\alpha \pi)}{\cos ^{2}(\alpha \pi)+\beta \sin ^{2}(\alpha \pi)}\right]
$$

with $\beta=[1+\exp (2 \pi i \ell q)]^{2} /[1-\exp (2 \pi i \ell q)]^{2}$. For the case $|A|<|D|$ Eq. (11) reduces to

$$
B=D-i(A D)^{1 / 2} \cdot \sin ^{2}(\pi \ell q) / \pi \text {. }
$$

This result, which can also be obtained by applying the boundary layer approach described earlier, indicates that the ion sound term makes a small stabilizing contribution to this class of modes.

Trapped-Ion Mode

For conditions approaching tokamak reactor requirements, the plasma is more collisionless and its parameters can fall within the regime where the dissipative trapped-ion mode is predicted to appear, namely $\nu_{1}^{\text {eff }}<\omega<v_{e}^{\text {eff }}$ with $\omega<\omega_{b i}^{T}<\omega_{b e}^{T}$ and $\omega \simeq \omega_{o}=\varepsilon 1 / 2 \omega_{*_{e}} / 2$. In estimating the diffusion arising from this instability the most common approach has been to examine the $\tilde{\tilde{E}} \times \underline{B}$ motion where $\underline{\tilde{E}}$ is the oscillatory electric field. This leads to the approximate result $D \simeq c^{2}|\tilde{E}|^{2} \gamma / B^{2} \omega^{2}$ with $\tilde{E}$ determined from the condition that the local density gradient be locally annihilated by the perturbations. Here we consider a model, similar to one first proposed by Kadomtsev and Pogutse [1], which includes the nonlinear $\underline{E} \times \underline{B}$ motion of the trapped particles, and analyze the following model equations for the trapped-particle densities $\mathrm{n}_{\mathrm{e}, \mathbf{i}}^{\mathrm{T}}$

$$
\partial n_{e, i}^{T} / \partial t+\left(c E \times B / B^{2}\right) \cdot \nabla n_{e, i}^{T}=-\nu_{e, i}^{e f f}\left[n_{e, i}^{T}-n_{o} \exp ( \pm e \phi / T)\right]
$$

together with the quasineutrality condition

$$
\mathrm{n}_{\mathrm{e}}^{\mathrm{T}}+\mathrm{n}_{\mathrm{o}} \exp (\mathrm{e} \phi / \mathrm{T})=\mathrm{n}_{\mathrm{i}}^{\mathrm{T}}+\mathrm{n}_{\mathrm{o}} \exp (-\mathrm{e} \phi / \mathrm{T}) \text {. }
$$

Since it is known from the linear theory of these modes that for suffi-. ciently weak temperature gradients the unstable spectrum is limited at short. wavelengths by ion Landau bounce-resonance damping, we include an additional. dissipative term of order $-\left(\omega^{4} / \omega_{b i}^{3}\right) n_{i}^{T}$ on the right of Eq. (13) for the ions to incorporate this important effect $[4,5]$.

The above equations are analyzed in the slab limit with the usual coordinates $r, \zeta-q \theta$ replaced by $x, y$. In addition, we transform to a reference frame moving in the $y$-direction with speed $\dot{v}_{*}=\omega_{0} / k_{y}$ and obtain the following nonlinear equation for the normalized potential, $\psi$

$$
\frac{\partial \psi}{\partial \tau}+\frac{\partial^{2} \psi}{\partial \xi^{2}}+\nu \psi+\alpha \frac{\partial^{4} \psi}{\partial \xi^{4}}+\frac{\partial \psi^{2}}{\partial \xi}+\beta\left(\frac{\partial \psi}{\partial \chi} \frac{\partial^{2} \psi}{\partial \xi^{2}}-\frac{\partial \psi}{\partial \xi} \frac{\partial^{2} \psi}{\partial \chi \partial \xi}\right)=0
$$

where $\psi=\left(\nu_{e}^{e f f} / \omega_{o} \varepsilon^{1 / 2}\right)(e \phi / T), \xi \xi=k_{y}\left(y-v_{*} t\right), x=k_{x} x, \tau=\gamma t=\left(\omega_{n}^{2} / \nu_{e_{j}}^{e f f}\right) t$,

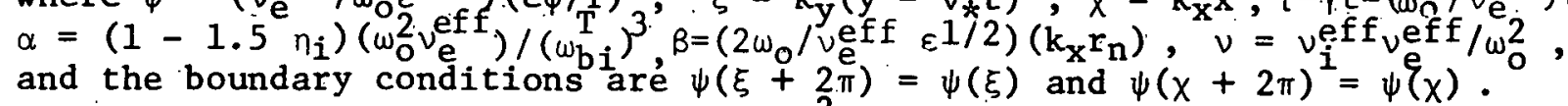
Here the nonlinear convective term, $\partial \psi^{2} / \partial \xi$, effectively "steepens" the , wave and provides a mechanism for transferring energy from long to short wavelengths which in turn can be ion Landau damped. The fourth derivative term is ion Landau damping, and comes from transforming $\omega^{4} / \omega_{\mathrm{bi}}^{3}$ to the moving frame.

We first consider the one-dimensional problem which results when the last term in Eq." (15) is negligible. For $\alpha \sim 1$ the system can be marginally stable, and saturation of the fluctuations is determined by a mode coupling calculation which yields the asymptotic solution 


$$
\psi_{\tau \rightarrow \infty}=\left(\gamma_{1} \gamma_{2}\right)^{1 / 2}\left[\sin \xi+\left(\left|\gamma_{1}\right| /\left|\gamma_{2}\right|\right)^{1 / 2} \sin (2 \xi)+\ldots\right]
$$

where $\gamma_{n}$ (growth rate of $n$th Fourier mode) $=n^{2}-\alpha n^{4}-v$ with $\gamma_{1}>0$ and $r_{2}<0$. For $0<\alpha \ll 1$, and neglecting $v$, the problem is solved by multiple scale length expansion (WKB) techniques giving the following steady state solution

$$
\Psi=n d(u)\left\{\left(c_{m}^{2} / 4\right)\left(k / k^{\prime}\right)^{2} \operatorname{sn}(u) \operatorname{cn}(u)+c_{m} \sin \left[\phi(\xi) / \alpha^{1 / 2}\right]\right\}
$$

where $u=(K \xi / \pi, k), c_{m}^{2}=4 k^{\prime 2} K(k) / \pi, k^{\prime}=\left(1-k^{2}\right)^{1 / 2}, K$ is the complete elliptic integral of the first kind, nd, sn, cn are Jacobi elliptic functions, and $k$ is determined by the appropriate WKB periodicity condition. Equation (17) represents a solitary wave solution, involving both long and short wavelength components.

Using Eq. (16) we find that near marginal stability $(\alpha \sim 1)$ the diffusion coefficient is

$$
D \simeq \frac{\varepsilon^{3 / 2} \omega_{o}^{2}}{\left(\nu_{e}^{e f f}\right)^{3}}\left(\frac{k_{y} c T}{e B}\right)^{2}\left|\gamma_{1} \gamma_{2}\right| .
$$

Far from marginal stability $(0<\alpha<1)$ many unstable modes can be present, and Eq. (17) is used to obtain

$$
D \simeq \frac{\varepsilon^{3 / 2}\left(\omega_{b i}^{T}\right)^{3}}{\left(\nu_{e}^{e f f}\right)^{4}}\left(\frac{k_{y} c T}{e B}\right)^{2} f(k)
$$

where $0 \leq f(k) \leq 1$. In Eq. (19), $k_{y}$ should be chosen between $\omega_{0}-v_{e}^{\text {eff }}$ and the longest wavelength not stabilized by ion collisions. Despite the fact that these expressions scale more pessimistically with temperature, Eqs. (18) and (19) predict less diffusion for parameters typical of next generation tokamaks than the commonly used estimate cited earlier. They are, of course, restricted to the case $\omega_{0} \ll \nu_{e}^{e f f}$.

In addressing the two-dimensional problem we have studied the nonlinear evolution of the fractional ion density fluctuation, $N=2 \psi$, by following it over long time intervals $(>1 / \gamma)$ with a computer code which solves. Eq. (15). The code advances in time $\mathrm{N}$, initially a random function of space, by a "leap-frog method" on a $64 \times 64 \mathrm{x}$-y grid, and has yielded solutions for various prescribed wavenumber spectra. For cases where the B-term in Eq. (15) is dominant, a class of analytic 2-D solutions have been found of the form $N \sim k_{x} x\left[1+b(t) \cos k_{y} y\right]$ where $b(t)$ is either an exponential growth or damping factor. The growth case corresponds to the presence of localized short wavelength "flutes" with wave vectors in the $y$-direction. This is in good agreement with numerical solutions which exhibits such behavior in regions where $-\partial N / \partial x$ is large.

A different type of nonlinear mechanism that can lower the amplitude of the prevailing electric field (and hence the diffusion) for the trappedion mode is one that arises from a velocity-space instability. Here the untrapped ions and all the electrons remain essentially Maxwellian, while the trapped-ion distribution develops approximately in the form

$$
f_{0}=F^{M}\left[1+\alpha \theta\left(2 \varepsilon v_{\perp}^{2}-v_{11}^{2}\right)\right]
$$


where $F^{M}$ is a Maxwellian, $\theta(x)$ is a step function, $\alpha=e \tilde{\phi}\left(1+T_{i} / T_{e}\right) / T_{i}(2 \varepsilon)^{\frac{1}{2}}$, and $\tilde{\phi}$ is the oscillatory low-frequency potential. Taking this for the trapped-ion equilibrium distribution function and considering electrostatic ion-cyclotron waves with $k_{1} v_{i} \gg \omega \sim \omega_{c i} \gg k_{11} v_{e}, \omega_{e}^{*}$, we obtain the following dispersion relation

$$
\hat{\gamma}+\dot{i} s g\left(k_{11}\right)+\hat{r} F(\Omega)=0
$$

where $\hat{\gamma}=k_{11} k_{1} \rho^{2}, \hat{r}=\left(\alpha / \pi^{1 / 2}\right) k_{1} \rho_{i}, \Omega=\left(1-\omega / \omega_{c i}\right)\left[k_{1} / k_{11}(2 \varepsilon)^{1 / 2}\right]$, $\mathrm{F}(\Omega)=\int_{-\infty}^{\infty} \mathrm{dxJ}_{1}(\mathrm{x}) /\left[\Omega-\mathrm{x}+i \varepsilon \mathrm{sg}^{\left(\mathrm{k}_{11}\right)}\right]$, and $\mathrm{sg}\left(\mathrm{k}_{11}\right)$ is the sign of $\mathrm{k}_{11}$. On those flux lines where $\alpha>0$, Eq. (21) gives unstable modes with a maximum growth rate, $\gamma_{\mathrm{HF}} \simeq 0.06 \varepsilon^{1 / 2} \alpha^{2} \omega_{\mathrm{ci}}$. This high frequency instability leads to a velocity-space scattering rate,

$$
\nu_{i}^{\text {eff }} \simeq 2\left(\gamma_{\mathrm{HF}} / \mathrm{k}_{\perp}^{2} \rho_{i}^{2}\right)_{\max } \simeq 0.014 \alpha^{4} \varepsilon^{1 / 2} \omega_{\mathrm{ci}} .
$$

After taking its spatial average we use this expression instead of the ion Coulomb collision frequency in the linear theory [4] to obtain the stability criterion, $x \nu$ eff $=10 \gamma_{0}$ where $\gamma_{0}=\left(1+1.4 n_{e}\right) \varepsilon^{2} \omega \omega_{e} /\left(1+T_{e} / T_{i}\right)^{2} v_{e}$ and $x$ is a number, typically of order unity, that is calculated in Ref. [4].

With the saturation amplitude, $\tilde{\phi}$, determined by the above, we estimate the diffusion from

$$
D \simeq\left(c^{2} / B^{2}\right)\left(\ell^{2} q^{2} \gamma_{L F} / r^{2} \omega_{L F}^{2}\right)<|\tilde{\phi}|^{2}>
$$

where $\omega_{L F}$ and $\gamma_{L F}$ are the frequency and growth: rate of the trapped-ion instability: This is the appropriate expression if it is less than the usual diffusion coefficient obtained from requiring destruction of local density gradients, namely, $D_{0} \simeq \gamma_{L F} / k_{\perp}^{2} \simeq 2 \gamma_{0} / k_{\perp}^{2}$. Hence, we can write

$$
D \simeq \begin{cases}D_{0}\left(\ell / \ell_{1}\right)^{3}, & \ell<\ell_{1} \\ D_{0}, & \ell>\ell_{1}\end{cases}
$$

where $\ell_{1} \simeq 0.5 \cdot\left[\left(1+\mathrm{T}_{i} / \mathrm{T}_{\mathrm{e}}\right)^{2} \omega_{c i} \nu_{\mathrm{e}} \mathrm{x} /\left(1+1.4 n_{\mathrm{e}}\right) \varepsilon^{3 / 2} \omega_{\star \mathrm{e}^{2}}^{2} \mathrm{q}^{4}\right]^{1 / 6}$. This indicates that the new high frequency saturation mechanism leads to a lower diffusion level for low toroidal mode numbers, $\ell$. However, in some situations the high frequency mode cannot be triggered, since it is found to be convective along the field line, and the finite scale length in tokamaks can therefore limit its amplification length. If we demand A e-folding lengths of the high-frequency wave in the tokamak trapping region (typically $A \simeq 10$ ), we find $D$ is modified to

$$
\mathrm{D} \simeq \begin{cases}\mathrm{D}_{0}\left[\left(\ell / l_{1}\right)^{3}+\left(l / l_{2}\right)^{2}\right], & \ell<\min \left(l_{1}, l_{2}\right) \\ \mathrm{D}_{0} & , \quad \ell>\min \left(l_{1}, l_{2}\right)\end{cases}
$$

where $\ell_{2}=\left(3 / A^{1 / 2}\right)\left(R / \rho_{i} q\right)^{1 / 2} \varepsilon^{-1 / 8}$. In order for the diffusion to be less than $D_{0}$, it is necessary that the high mode number cutoff, $\ell_{m}$, obtained by maximizing $\gamma_{L F} / k_{1}^{2}$ in Ref. [5], be less than $\ell_{1}$ or $\ell_{2}$. Unfortunately, for typical parameters $\left(B=50, \mathrm{kG}, \mathrm{T}=10 \mathrm{KeV}, \varepsilon=1 / 4, q=3, \eta_{i}=\eta_{e}=1 / 2\right.$, $\left.\eta=10^{14} \mathrm{~cm}^{-3}\right) \ell_{\mathrm{m}} \geq \ell_{1}>\ell_{2}$ with the ratio $\ell_{\mathrm{m}} / \ell_{1}$ quite insensitive to changes in $T$ and $n$. Hence, in practice the trapped particle diffusion is not appreciably reduced by the high frequency saturation process. 


\section{REFERENCES}

* Supported by USAEC Contracts $\operatorname{Ar}(11-1)-3073$ and AT (40-1)-4478.

+ Now at Lawrence Livermore Laboratory, Livermore, California.

$\ddagger \quad$ Also at Institute for Advanced Study, Princeton, New Jersey.

[1] KADOMSTSEV, B. B., POGUTSE, 0. P., Nuc1. Fusion 11, $67(1971)$.

[2] GaleEV, A. A., Third Int. Symp. on Toroidal Plasma Confinement, Garching, March 26-30, 1973 (proc. to be pub.).

[3] GLADD, N. T., HORTỌ, W., Jr., Phys. Fluids 16, 879(1973).

[4] ROSEnbluth, M. N., ROSS, D. W., KOStOMAROV, D. P., Nucl. Fusion 12, 3(1972).

[5] TANG, W. M., Nucl. Fusion 13, 883(1973). 


\section{LE G A L N O T I C E}

This report was prepared as an account of Government sponsored work. Neither the United States, nor the Commission, nor any person acting on behalf of the Commission:

A. Makes any warranty or representation, express or implied, with respect to the accuracy, completeness, or usefulness of the information contained in this report, or that the use of any information, apparatus, method, or process disclosed in this report may not infringe privately owned rights; or

B. Assumes any liabilities with respect to the use of, or for damages resulting from the use of any information, apparatus, method, or process disclosed in this report.

As used in the above, "person acting on behalf of the Commission" includes any employee or contractor of the Commission to the extent that such employee or contractor prepares, handles or distributes, or provides access to, any information pursuant to his employment or contract with the Commission. 